\title{
m. \\ ADIPOCYTE DYSFUNCTION IN PEDIATRIC OBESITY
}

\section{Iuliana Gherlan, Andra Caragheorgheopol, Suzana Vlădoiu, Adriana Pădure, Florin Alexiu, Sorina Șchipor}

"C.I.Parhon" National Institute of Endocrinology, Bucharest, Romania

Background. Obesity is associated with adipocyte dysfunction, characterized by an impaired secretion of adipokines, which leads to a systemic inflammatory status, insulin resistance and atherogenesis.

Aim. To characterize adipokines serum profile in a group of obese children and adolescents.

Method. A case control study comparing 102 obese children (BMI $\geq 95^{\text {th }}$ percentile) (aged 10-18 years) to a group of 43 healthy controls matched for age and pubertal status.

Adiponectin, leptin, resistin, A-FABP and lipocalin-2 were measured using ELISA method and were correlated with traditional clinical and biochemical biomarkers of the metabolic syndrome (BMI, waist circumference, blood pressure, fasting glycaemia and insulinemia, lipids profile). The BMI, waist circumference (WC), systolic and diastolic blood pressure (BP) were adjusted for age, sex and height according to WHO growth charts and the NHANES references. HOMA-index was calculate using the formula: a jeun glycaemia (mg/dl) ${ }^{*} a$ jeun insulinemia $\mu U I / m I / 405$; insulin resistance summary score (IRS) was calculated by adding the quartiles for systolic blood pressure, a jeun glycaemia, insulinemia and serum triglycerides (analyzed ascending) and serum HDL-cholesterol (analyzed descending).

Results. Obese children had significantly higher waist circumferences, BP and serum levels of triglycerides and significantly lower serum levels of HDL-cholesterol (table 1). All insulin resistance biomarkers (a jeun insulinemia, HOMA-index and IRS) were significantly higher in the obese group compared to the control group (figure 1).

\begin{tabular}{|c|c|c|c|}
\hline \multirow{2}{*}{$\begin{array}{l}\text { Parameter } \\
\text { BMI (Kg/sqm) }\end{array}$} & \multicolumn{2}{|c|}{ Obese group $(n=102)$ Control group $(n=43)$} & \multirow{2}{*}{$\begin{array}{l}p \\
p<0.01\end{array}$} \\
\hline & $31.23 \pm 5.09$ & $19.14 \pm 2.71$ & \\
\hline Z Score for BMI & $2.17 \pm 0.3$ & $-0.21 \pm 0.96$ & $p<0.01$ \\
\hline$W C(\mathrm{~cm})$ & $101.57 \pm 11.29$ & $77.75 \pm 10.43$ & $p<0.01$ \\
\hline Systolic BP $(\mathrm{mmHg})$ & $123.09 \pm 13.26$ & $102.67 \pm 9.09$ & $p<0.01$ \\
\hline Systolic BP percentile & $80.77 \pm 21.61$ & $30.69 \pm 22.72$ & $p<0.01$ \\
\hline Diastolic BP $(\mathrm{mmHg})$ & $73.38 \pm 10.34$ & $62.09 \pm 7$ & $p<0.01$ \\
\hline Diastolic BP percentile & $72.55 \pm 22.31$ & $42.73 \pm 18.86$ & $p<0.01$ \\
\hline Glycaemia (mg/dl) & $79,87 \pm 11,07$ & $83,68 \pm 8,22$ & 0,058 \\
\hline Total-cholesterol (mg/dl) & $166,29 \pm 36,76$ & $160,89 \pm 27,23$ & 0,392 \\
\hline HDL - cholesterol (mg/dl) & $44,94 \pm 9,55$ & $51,72 \pm 10,48$ & 0,02 \\
\hline LDL-cholesterol (mg/dl) & $102,73 \pm 30,30$ & $100 \pm 31,93$ & 0,69 \\
\hline Triglycerides (mg/dl) & $108,01 \pm 58,23$ & $80,48 \pm 37,6$ & 0,005 \\
\hline
\end{tabular}

The serum levels of leptin, resistin, AFABP and lipocalin-2 were significantly higher while the adiponectin serum levels were significantly lower in the obese group compared to the control group (table 2, figure 2).

All the adipokines were significantly correlated (in univariate analysis) to $\mathrm{Z}$ score for BMI (table 3).

Adiponectin, leptin, AFABP and resistin were significantly correlated to systolic and diastolic blood pressure (negative correlation for adiponectin, positive correlations for others adipokines); there was also a parallel variation of the previous mentioned adipokines and HDL-cholesterol, positive for adiponectin and negative for leptin, resistin and AFABP (table 3).

Adiponectin, leptin and A-FABP also correlated with various biomarkers of insulin resistance (table 3); the correlation between leptin serum levels and fasting insulinemia was kept in multivariate analysis.

\section{In multivariate analysis:}

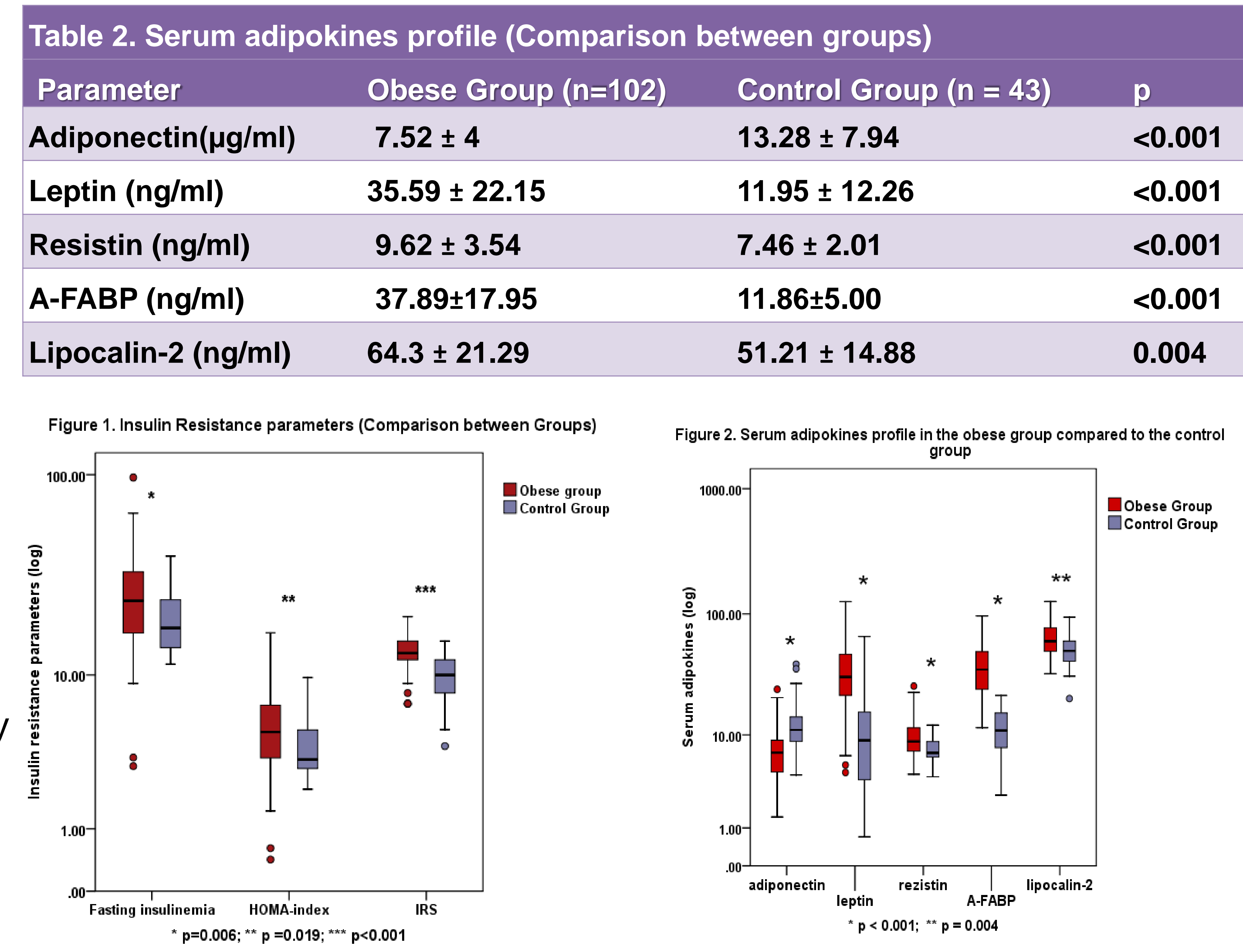

- Adiponectin was negatively correlated to waist circumference and serum A-FABP Figur 3. correation between serum adiponectin and wasts circumference ( $R$ coefficient $=-0.627, p<0.001)$ (fig. 3,4);

- Leptin was positively correlated to waist circumference and fasting insulinemia $(R$

coefficient $=0.682, p<0.001$ ) (fig. 5,6 );
A-FABP was positively correlated to $Z$ score for BMI and adiponectin (R coefficient $=0,700, p<0,001$ ) (fig. 4,7);

- Resistin was positively correlated only to lipocalin-2 ( $R$ coefficient $=0.514, p<<$ 装 10000 0.001) (fig.8).

Conclusions. Pediatric obesity is already associated with an altered production of adipokines, which participates in the pathogenesis of obesity-associated comorbidities.
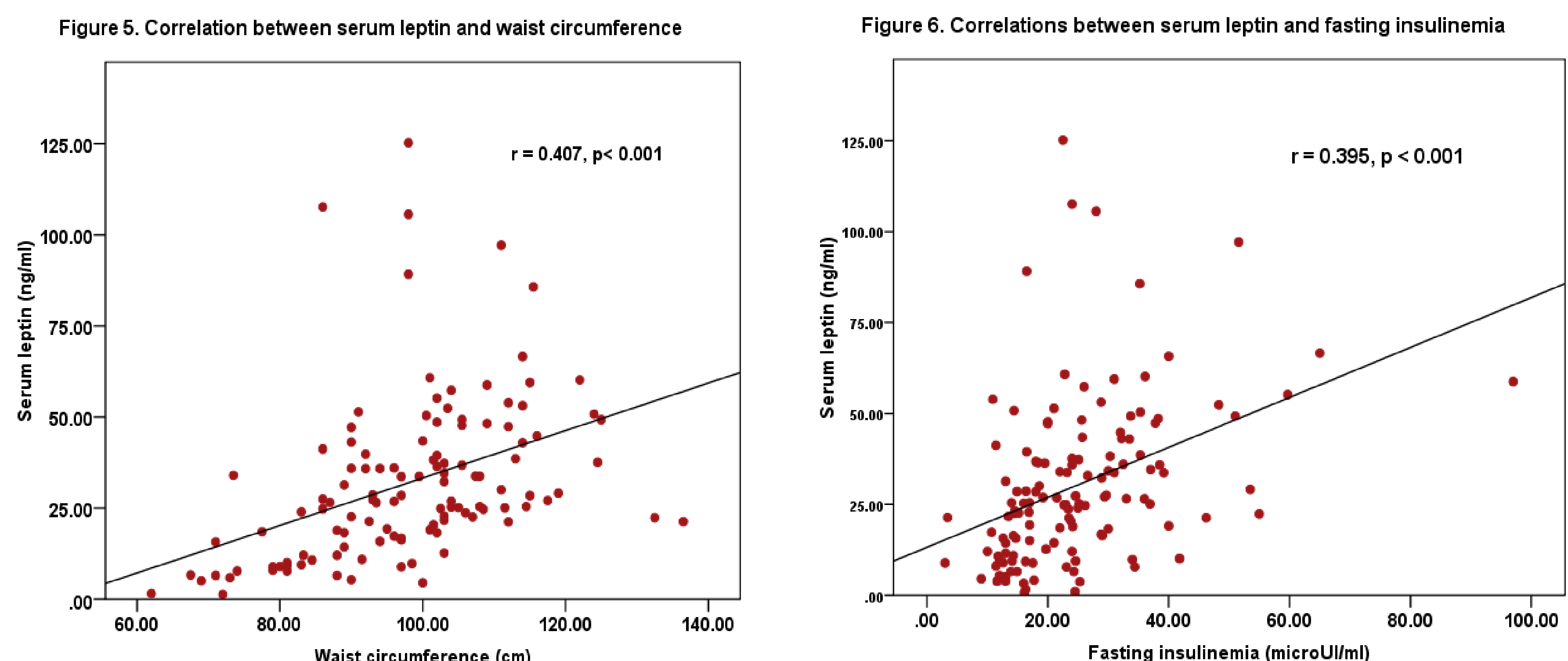

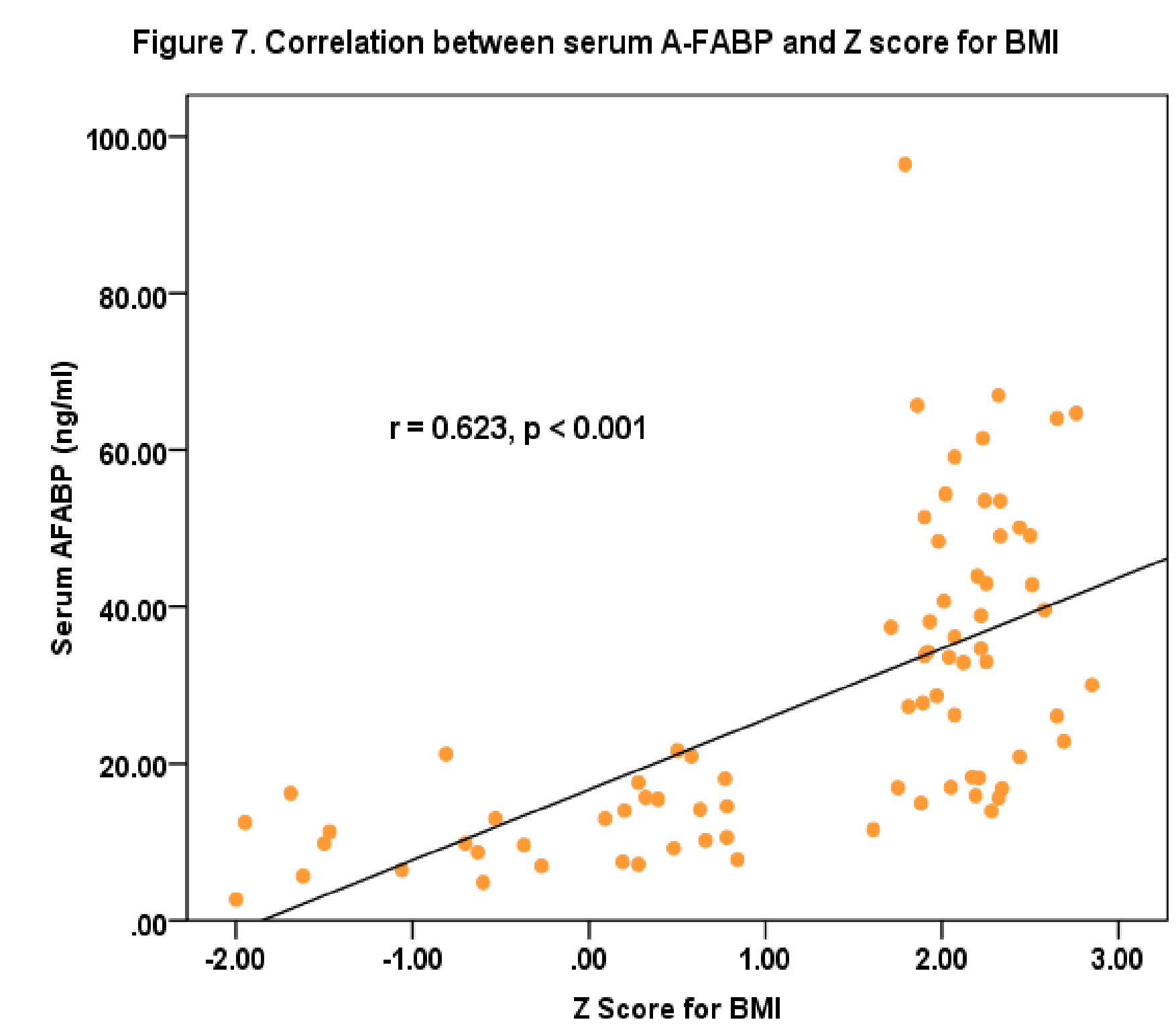

Lipocalin-2 $0,295, p=0,009$

$0,510, p<0,001$

$0,395 p<0,001$

$0,353 p<0,001$

$0,460 p<0,001$
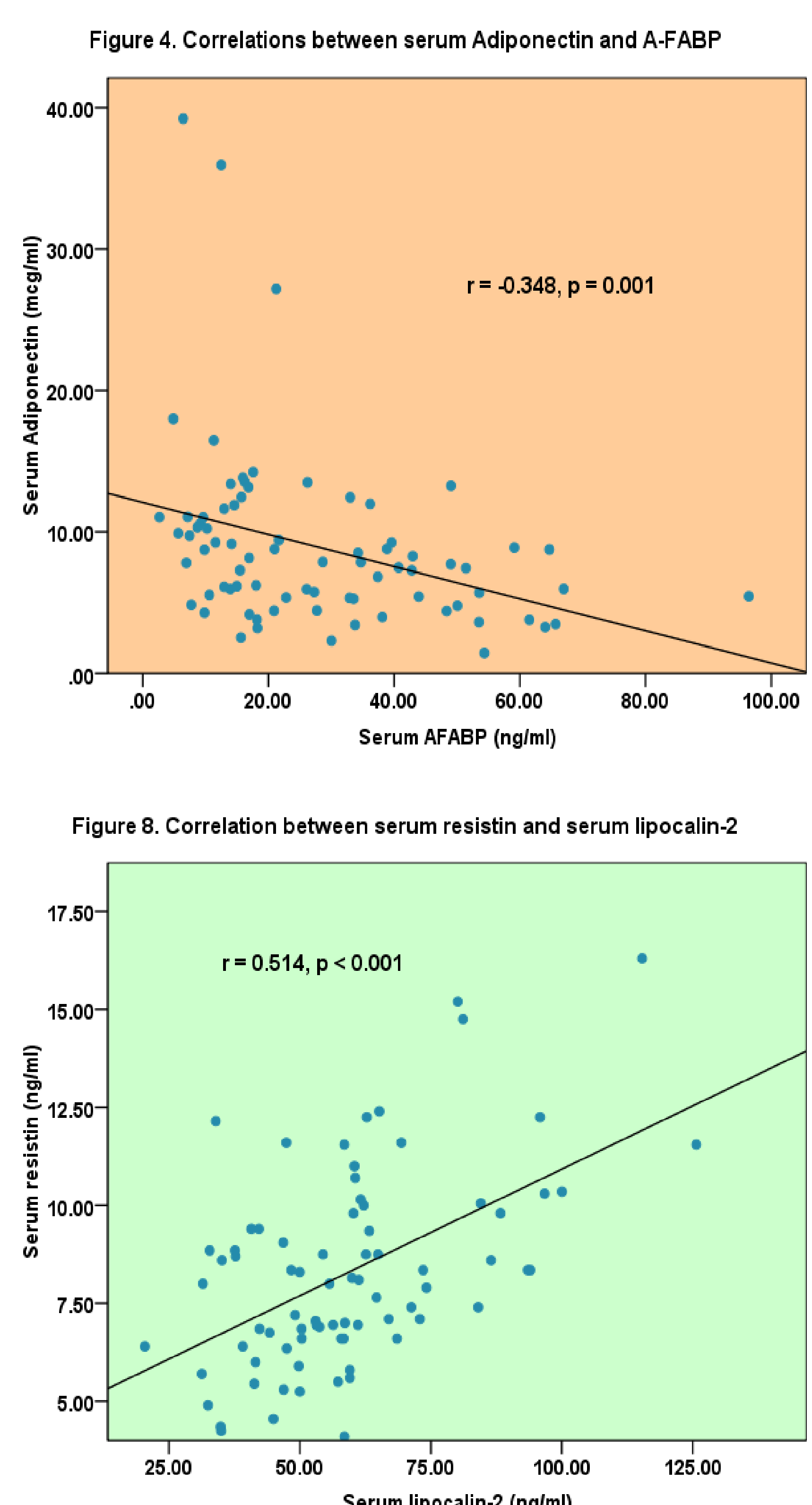